\title{
Spectral Analysis of Extended Consensus Algorithms for Multiagent Systems
}

\author{
Sebastian van de Hoef, Dimos V. Dimarogonas and Panagiotis Tsiotras
}

\begin{abstract}
We analyze an extension of the well-known linear consensus protocol for agents moving in two dimensions, where the standard consensus feedback is multiplied with a rotation matrix. This leads to a richer family of trajectories, and if only the new feedback term is applied, periodic solutions emerge. For special configurations of the controller gains, the form of the system trajectories is given in terms of the eigenvalues and eigenvectors of the closed-loop system matrix. We characterize the resulting closed-loop trajectories for specific choices of the controller gains and of the communication graph topology. Furthermore, the control strategy is extended to agents with double integrator dynamics. It is shown that stability is achieved with sufficiently large velocity feedback. The effect of this feedback on the overall system performance is further investigated. We finally provide simulations to illustrate the theoretical results.
\end{abstract}

\section{INTRODUCTION}

In recent years, consensus algorithms have become the cornerstone for decentralized control strategies. This class of algorithms can be applied when a number of agents need to agree on a commonly accepted consensus value, while each agent only has access to information from a subset of the other agents. Consensus algorithms often offer the building blocks of more complex control schemes.

The majority of the work on consensus algorithms focuses on the asymptotic behavior of the system [1]. The results reported in literature address various agent dynamics, and different consensus points with respect to the initial conditions are considered [2].

Another topic, that is closely related to the results in this paper, is vehicle coordination. Classical objectives in this context are related to flocking, swarming and formation. The reader may refer to [3], [4] for more information. Finally, we mention the related work on circumnavigation, see for instance [5], [6].

The work presented in this paper analyzes and extends the consensus feedback recently presented in [7]. Therein, the authors propose an extension of the classical linear continuous-time consensus for plants with single integrator dynamics. In contrast to standard linear consensus strategies, where the feedback action is along the relative line-ofsight of each pair of agents, in that work an additional feedback term, which is perpendicular to this line-of-sight

The first two authors are with the ACCESS Linnaeus Center, School of Electrical Engineering, KTH Royal Institute of Technology, SE-100 44, Stockholm, Sweden. $\{$ shvdh, dimos $\} a k t h . s e$. Their work was supported by the Swedish Research Council (VR). The second author is also affiliated with the KTH Centre for Automomous Systems. The third author is with the School of Aerospace Engineering, Georgia Institute of Technology, Atlanta, GA 30332-0150, USA, tsiotras@gatech. edu. His work was supported by AFOSR award no. FA9550-13-1-0029. direction, is considered. This new feedback strategy can produce consensus points outside the convex hull of the initial agent positions. Since the paths taken by the agents show a great variety, depending on the feedback gain coefficients, initial conditions and graph structure, this paper focuses on analyzing the transient behavior of the system for single integrator dynamics. While the standard consensus feedback introduces a potential field that guides the trajectories, the approach in [7] introduces a gyroscopic/non-potential field. If only "gyroscopic" feedback is applied, the system exhibits periodic solutions having intricate patterns in the state space [8]. Furthermore, we extend the control strategy to double-integrator dynamics and analyze the asymptotic behavior of the resulting system.

A similar feedback strategy is proposed in [9], which can be seen as a special case of the feedback law proposed in [7]. For the single integrator part, the work presented in [9] focuses more on the asymptotic behavior, while we focus on the transient behavior and in particular on sustained oscillations. For the part on double integrators, we consider relative instead of absolute damping.

After some preliminaries in Section II, we analyze in Section III the system trajectories of the control strategy proposed in [7] for a special configuration of the weight matrices. We focus, in particular, on the case in which the system exhibits sustained oscillations. We give a number of results on the closed-loop trajectories for the general case, and for the special case where all weights are equal and the graph Laplacian is a circulant matrix. In Section V, we propose an extension of the control strategy to double integrator dynamics. We give a sufficient condition for convergence and quantitatively analyze the influence of the velocity feedback term. Finally, in Section VI, we provide simulation results for single- and double-integrator dynamics to illustrate the theory, and in Section VII we summarize the contributions of the paper and offer some directions for future work.

\section{SySTEM LAYOUT AND PRELIMINARIES}

Let $\mathbf{I}$ denote the identity matrix and let $\mathbf{0}$ denote the zero matrix, with dimension apparent from the context. Let 1 denote the vector of all ones and let $(\cdot)^{\mathrm{T}}$ denote the transpose of a vector. All vectors will be assumed to be column vectors, $\mathbf{A}>0$ denotes that the matrix $\mathbf{A}$ is positive definite, and $\jmath$ denotes the imaginary unit.

A framework similar to the one presented in [7] is considered. To this end, consider $N$ agents, each with state vector $\mathbf{x}_{i} \in \mathbb{R}^{2}$ for agents $i \in\{1, \ldots, N\}$, having single integrator dynamics, as follows $\dot{\mathbf{x}}_{i}=\mathbf{u}_{i}$, with $\mathbf{u}_{i} \in \mathbb{R}^{2}$. The agent 
connections are encoded via an undirected graph $\mathcal{G}=(\mathcal{V}, \mathcal{E})$ where $\mathcal{V}$ is the set of nodes and $\mathcal{E}=\{(i, j) \in \mathcal{V} \times \mathcal{V}\}$ is the set of edges. Since $\mathcal{G}$ is undirected, $(i, j) \in \mathcal{E}$ if and only if $(j, i) \in \mathcal{E}$. The graph-theoretic notions used below are largely adopted from [10]. We will assume that $\mathcal{G}$ is static and connected. Every agent corresponds to a node in $\mathcal{V}$, and an edge between two agents, say $i$ and $j$, implies that the corresponding agents are mutually able to measure the relative vector $\mathbf{x}_{i}-\mathbf{x}_{j}$.

Every agent (index $i$ ) uses the state difference to each of its neighbors (index $j$ ) $\mathbf{z}_{\text {agent }, i j}=\mathbf{x}_{j}-\mathbf{x}_{i}$ to compute its control action. Consequently, $\mathbf{z}_{\text {agent }, i j}=-\mathbf{z}_{\text {agent }, j i}$. In order to globally describe the system behavior for every edge $(i, j)$ in $\mathcal{G}$, either the error variable $\mathbf{z}_{i, j}=\mathbf{z}_{k}=\mathbf{z}_{\text {agent }, i j}$ or $\mathbf{z}_{i, j}=\mathbf{z}_{k}=-\mathbf{z}_{\text {agent }, i j}$ is chosen. The orientation of $\mathbf{z}_{k}$ will not play a role in the analysis and is hence arbitrary. This asymmetry can be described by a directed graph $\mathcal{G}_{\mathrm{d}}$, where agent $i$ is the head of the edge between nodes $i$ and $j$ if $\mathbf{z}_{i, j}=-\mathbf{z}_{\text {agent }, i j}$ and the tail of the edge if $\mathbf{z}_{i, j}=\mathbf{z}_{\text {agent }, i j}$. The matrix $D \in \mathbb{R}^{N \times M}$ will denote the incidence matrix of $\mathcal{G}_{\mathrm{d}}$ [10]. All $\mathbf{z}_{k}$ are stacked together to form the vector $\mathbf{z}=\left[\begin{array}{llll}\mathbf{z}_{1}^{\mathrm{T}} & \mathbf{z}_{2}^{\mathrm{T}} & \ldots & \mathbf{z}_{M}^{\mathrm{T}}\end{array}\right]^{\mathrm{T}} \in \mathbb{R}^{2 M}$. According to [7], the relation

$$
\mathbf{z}=\left(\mathbf{D}^{\mathrm{T}} \otimes \mathbf{I}_{2}\right) \mathbf{x}
$$

holds, where $\mathbf{I}_{2}$ is the $2 \times 2$ identity matrix and $\mathbf{x}=$ $\left[\begin{array}{llll}\mathbf{x}_{1}^{\mathrm{T}} & \mathbf{x}_{2}^{\mathrm{T}} & \ldots & \mathbf{x}_{N}^{\mathrm{T}}\end{array}\right]^{\mathrm{T}} \in \mathbb{R}^{2 N}$. In this paper we are concerned with the analysis of the trajectories generated by the control law introduced in [7], which is of the form

$$
\mathbf{u}=-\left(\boldsymbol{\Gamma D} \otimes \mathbf{I}_{2}\right) \mathbf{z}+(\mathbf{B D} \otimes \mathbf{S}) \mathbf{z}
$$

with $\boldsymbol{\Gamma}=\operatorname{diag}(\gamma) \in \mathbb{R}^{N \times N}$, where $\gamma \in \mathbb{R}^{N}$ and $\mathbf{B}=$ $\operatorname{diag}(\boldsymbol{\beta}) \in \mathbb{R}^{N \times N}$ where $\boldsymbol{\beta} \in \mathbb{R}^{N}$, and where $\mathbf{S}=\left[\begin{array}{cc}0 & 1 \\ -1 & 0\end{array}\right]$. The vector $\mathbf{u}$ in (2) is a stack vector of all agent inputs, namely, $\mathbf{u}=\left[\begin{array}{llll}\mathbf{u}_{1}^{\mathrm{T}} & \mathbf{u}_{2}^{\mathrm{T}} & \ldots & \mathbf{u}_{N}^{\mathrm{T}}\end{array}\right]^{\mathrm{T}} \in \mathbb{R}^{2 N}$. Combining (1) and (2) yields

$$
\dot{\mathbf{x}}=\left(-(\mathbf{\Gamma L}) \otimes \mathbf{I}_{2}+(\mathbf{B L}) \otimes \mathbf{S}\right) \mathbf{x}=\mathbf{\Theta} \mathbf{x},
$$

where $\mathbf{L}=\mathbf{D} \mathbf{D}^{\mathrm{T}}$ is the Laplacian of $\mathcal{G}$ [10]. In terms of state differences, we have the corresponding equation

$$
\dot{\mathbf{z}}=\left(-\left(\mathbf{D}^{\mathrm{T}} \boldsymbol{\Gamma D}\right) \otimes \mathbf{I}_{2}+\left(\mathbf{D}^{\mathrm{T}} \mathbf{B D}\right) \otimes \mathbf{S}\right) \mathbf{z}=\boldsymbol{\Theta}_{z} \mathbf{z} .
$$

Equations (3) and (4) represent autonomous, linear systems in state space form.

\section{Trajectories With Particular Gain Matrices}

In this section we analyze (3) for the case where the gain matrices $\boldsymbol{\Gamma}$ and $\mathbf{B}$ are proportional. Furthermore, we analyze the solution when $\boldsymbol{\Gamma}=\mathbf{0}$. When $\boldsymbol{\Gamma}$ and $\mathbf{B}$ are proportional this means that $\boldsymbol{\Gamma}=\alpha_{\boldsymbol{\Gamma}} \mathbf{M}, \mathbf{B}=\alpha_{\mathbf{B}} \mathbf{M}$ for some $\alpha_{\boldsymbol{\Gamma}}, \alpha_{\mathbf{B}} \in$ $\mathbb{R}, \mathbf{M} \in \mathbb{R}^{N \times N}$ diagonal. This constraint in the choice of weights allows us to express the eigenvalues and eigenvectors of the $2 N \times 2 N$ matrix $-(\mathbf{\Gamma L}) \otimes \mathbf{I}_{2}+(\mathbf{B L}) \otimes \mathbf{S}$ in terms of the smaller $N \times N$ matrix $\mathbf{M L}$. The matrix $\mathbf{M L}$ is a weighted version of the graph Laplacian of $\mathcal{G}$. We call ML a scaled Laplacian matrix of $\mathcal{G}$.
Lemma 1. Let $\boldsymbol{\Gamma}=\alpha_{\boldsymbol{\Gamma}} \mathbf{M}, \mathbf{B}=\alpha_{\mathbf{B}} \mathbf{M}$ with $\alpha_{\boldsymbol{\Gamma}}, \alpha_{\mathbf{B}} \in \mathbb{R}$ $\mathbf{M} \in \mathbb{R}^{N \times N}$ diagonal. Let $\mathbf{v} \in \mathbb{R}^{N}$ be an eigenvector of ML with corresponding eigenvalue $\lambda \in \mathbb{R}$. Then, the vector $\overline{\mathbf{v}}=\mathbf{v} \otimes[1 \pm \jmath]^{\mathrm{T}} \in \mathbb{C}^{2 N}$ is an eigenvector of the matrix $\boldsymbol{\Theta}=-(\boldsymbol{\Gamma L}) \otimes \mathbf{I}_{2}+(\mathbf{B L}) \otimes \mathbf{S}$ with corresponding eigenvalue $\left(-\alpha_{\boldsymbol{\Gamma}} \pm \alpha_{\mathbf{B}} \jmath\right) \lambda$.

Proof. First, it is easy to show (see, for instance [7]), that the eigenvalues $(\lambda)$ and eigenvectors $(\mathbf{v})$ of $\mathbf{B L}$ are real. Let now $\eta_{i j}$ be the elements of the matrix BL. Assuming $\boldsymbol{\Gamma}=\alpha_{\boldsymbol{\Gamma}} \mathbf{M}, \mathbf{B}=\alpha_{\mathbf{B}} \mathbf{M}$, we obtain for the eigenvalues and eigenvectors of the matrix $\Theta$ as follows $\boldsymbol{\Theta} \overline{\mathbf{v}}=\left(-\left(\alpha_{\boldsymbol{\Gamma}} \mathbf{M} \otimes \mathbf{I}_{2}+\alpha_{\mathbf{B}} \mathbf{M} \otimes \mathbf{S}\right)\left(\mathbf{v} \otimes\left[\begin{array}{c}1 \\ \pm \jmath\end{array}\right]\right)=\right.$ $\left(\mathbf{M} \otimes\left(-\alpha_{\boldsymbol{\Gamma}} \mathbf{I}_{2}+\alpha_{\mathbf{B}} \mathbf{S}\right)\right)\left(\mathbf{v} \otimes\left[\begin{array}{c}1 \\ \pm \jmath\end{array}\right]\right)=\left(-\alpha_{\boldsymbol{\Gamma}} \pm \alpha_{\mathbf{B}} \jmath\right) \lambda \overline{\mathbf{v}}$ The last equation shows that $\left.\left(-\alpha_{\boldsymbol{\Gamma}} \pm \alpha_{\mathbf{B}}\right\}\right) \lambda$ is an eigenvalue of $\Theta$ with corresponding eigenvector $\overline{\mathbf{v}}$.

From Lemma 1, we gain insight of the effect of $\alpha_{\boldsymbol{\Gamma}}$ and $\alpha_{\mathbf{B}}$ on the system trajectories. Specifically, $\alpha_{\boldsymbol{\Gamma}}$ is proportional to the real and $\alpha_{\mathbf{B}}$ is proportional to the imaginary part of the eigenvalues of $\boldsymbol{\Theta}$. If $\alpha_{\mathbf{B}}=0$ we have the "ordinary" consensus control with all the eigenvalues of $\Theta$ being real. For $\alpha_{\boldsymbol{\Gamma}}=0$, all non-zero eigenvalues of $\boldsymbol{\Theta}$ lie on the imaginary axis. Hence, the system exhibits sustained oscillations, which could be used, for instance, for decentralized orbit pattern generation.

In the case where $\alpha_{\boldsymbol{\Gamma}}=0$ and $\mathrm{ML}$ is diagonalizable, we can visualize the system trajectories as superpositions of circular motions. Since the matrix $\Theta$ has real entries, its eigenvalues appear in complex conjugate pairs. The complex argument of the odd and even entries of the eigenvectors differ by $\pm \pi / 2$, and hence if $\mathrm{ML}$ is diagonalizable, the trajectories of the agents are superpositions of circular motions. According to Lemma 1, each conjugate pair of eigenvectors of $\Theta$ causes a circular motion with angular frequency equal to the eigenvalue $\lambda$ and radius related to the elements of $\mathbf{v}$.

Theorem 1. Let $\boldsymbol{\Gamma}=\mathbf{0}$ and $\mathrm{BL}$ be diagonalizable. Let $\mathcal{A}$ be the set of all eigenvectors $\mathbf{v}$ corresponding to the non-zero eigenvalues $\lambda_{\mathbf{v}} \neq 0$ of $\mathbf{B L}$. Then, the solution of (3) is given by $\mathbf{x}(t)=\overline{\mathbf{x}}+\sum_{\mathbf{v} \in \mathcal{A}}\left(\left|a_{\mathbf{v}}\right|\left(\cos \left(\lambda_{\mathbf{v}} t+\arg \left(a_{\mathbf{v}}\right)\right)\left(\mathbf{v} \otimes\left[\begin{array}{ll}1 & 0\end{array}\right]^{\mathrm{T}}\right)+\right.\right.$ $\left.\sin \left(\lambda_{\mathbf{v}} t+\arg \left(a_{\mathbf{v}}\right)\right)\left(\mathbf{v} \otimes\left[01^{\mathrm{T}}\right)\right)\right)$, where $\overline{\mathbf{x}} \in \mathbb{R}^{2 N}, a_{\mathbf{v}} \in \mathbb{C}$ are constants that are determined by the initial conditions.

Proof. If $\boldsymbol{\Gamma}=\mathbf{0}$ and $\mathbf{B L}$ is diagonalizable the solutions of (3) are $\mathbf{x}(t)=\sum_{i=1}^{2 N} a_{i} \overline{\mathbf{v}}_{i} \exp \left(\lambda_{i} t\right)$, where $\overline{\mathbf{v}}_{i}$ is the $i$ th eigenvector of $\Theta$ with eigenvalue $\lambda_{i}$ and $a_{i} \in \mathbb{C}$ is determined by the initial conditions. According to Lemma 1 with $\mathbf{M}=\mathbf{B}$, $\alpha_{\boldsymbol{\Gamma}}=0, \alpha_{\mathbf{B}}=1$, the eigenvalues and eigenvectors of $\boldsymbol{\Theta}$ are given by the eigenvalues and eigenvectors of BL according to $\lambda_{i}= \pm \lambda_{\mathbf{v}}$ and $\overline{\mathbf{v}}_{i}=\mathbf{v} \otimes\left[\begin{array}{ll}1 & \pm \jmath\end{array}\right]^{\mathrm{T}}$. The eigenvectors of BL with eigenvalue 0 give the constant part of the solution $\overline{\mathbf{x}}$, which must be real valued since $\Theta$ is real valued. The even positions in $\overline{\mathbf{v}}_{i}$ are phase-shifted by $\pi / 2$ with respect to the odd positions. Applying Euler's formula proves the 
theorem.

The center of the circular motions of the agents is $\overline{\mathbf{x}}$. The radius of the circular motion of the $i$ th agent, introduced by an eigenvector $\mathbf{v}$, is proportional to the $i$ th element of the eigenvector, and the angular frequency is determined by the corresponding eigenvalue $\lambda$. The radii are scaled by the magnitude $\left|a_{\mathbf{v}}\right|$, and the phase of the circular motion is determined by $\arg \left(a_{\mathbf{v}}\right)$. The initial conditions of (3) determine $\overline{\mathbf{x}}$ and $a_{\mathbf{v}}$. They can be chosen, for instance, in such a way that only a few of modes are present in the solution, which allows for the design of desired motion patterns.

Corollary 1. Given $\overline{\mathbf{x}}$ and $a_{\mathbf{v}}$ for $\mathbf{v} \in \mathcal{A}$ as in Theorem 1, the initial conditions $\mathbf{x}(0)$ are $\mathbf{x}(0)=\overline{\mathbf{x}}+$ $\sum_{\mathbf{v} \in \mathcal{A}}\left(\left|a_{\mathbf{v}}\right|\left(\cos \left(\arg \left(a_{\mathbf{v}}\right)\right)\left(\mathbf{v} \otimes\left[\begin{array}{ll}1 & 0\end{array}\right]^{\mathrm{T}}\right)+\sin \left(\arg \left(a_{\mathbf{v}}\right)\right)(\mathbf{v} \otimes\right.\right.$ $\left.\left.\left.\left[\begin{array}{ll}0 & 1\end{array}\right]^{\mathrm{T}}\right)\right)\right)$.

Proof. The result follows from setting $t=0$ in Theorem 1 .

With Theorem 1 and Corollary 1, we have a direct link between the system trajectories, the initial conditions, and the eigenvalues and eigenvectors of BL. This motivates us to further study the eigenvalues and eigenvectors of scaled Laplacians.

\section{Eigenvalues And Eigenvectors of ScAled LAPLACIANS}

The results in Section III motivate further investigation of the eigenvalues and eigenvectors of scaled Laplacian matrices. Recall that, by "scaled Laplacians," we mean matrices of the form BL where $\mathbf{B}$ is a diagonal matrix, with diagonal entries $\beta_{1}, \ldots, \beta_{N}$, and $\mathbf{L}$ is a graph Laplacian. We will first give some useful properties for a general matrix BL. Then we give more specific results for the case of special communication graphs and with $\mathbf{B}=\alpha \mathbf{I}$.

\section{A. General Graph Structure and Weights}

An important feature for the analysis of consensus algorithms is the property of the graph Laplacian that the only eigenvector with eigenvalue zero is $\mathbf{1}$. First, we show that this property holds even for scaled Laplacians, if at most one of the weights $\beta_{i}$ is zero. In order to show this result we first provide the following lemma.

Lemma 2. Let $m$ be the number of zero diagonal entries of $\mathbf{B}$. If $\mathcal{G}$ is connected, then the rank of $\mathbf{B L}$ is $N-1$ for $m=0$ and $m=1$ and $N-m$ for $m>2$.

Proof. Recall that $\mathbf{L}$ has rank $N-1$ if $\mathcal{G}$ is connected [1]. The multiplication with the matrix $\mathbf{B}$ scales the row vectors and therefore it does not change the rank of the matrix if there are no zero entries in $\boldsymbol{\beta}$. Hence, any set of $N-1$ row vectors of $\mathbf{L}$ is linearly independent. Therefore, setting one entry in $\boldsymbol{\beta}$ to zero will not change the rank of BL. Any additional zero entry in $\boldsymbol{\beta}$ will decrease the rank by one as it replaces one linearly independent row vector by a row of zeros.
With the previous lemma we can prove Theorem 2:

Theorem 2. If $\beta_{i}=0$ for at most one $i \in \mathcal{V}$, and $\mathcal{G}$ is connected, then the null space of $\mathrm{BL}$ is one-dimensional and it is spanned by the vector 1.

Proof. Since the rank of BL in this case is $N-1$, there is only one linearly independent solution, say $\mathbf{v}$, to the equation $\mathbf{B L v}=0$. Since the vector $\mathbf{1}$ has the property $\mathbf{L} \mathbf{1}=\mathbf{0}$ (see [10]) 1 spans the whole null-space of BL.

From the signs of the diagonal elements of $\mathbf{B}$, we can infer the signs of the eigenvalues of BL. Considering Theorem 1, we see that the sign of $\lambda_{\mathbf{v}}$ determines the direction of rotation (clockwise/counter-clockwise) of the corresponding mode. Therefore, insight into the relation of the weights and the signs of the eigenvalues can provide valuable information for trajectory design purposes.

Theorem 3. If $\boldsymbol{\beta}$ has $m$ positive entries and $N-m$ negative entries, then $\mathrm{BL}$ has $m$ eigenvalues $\lambda$ with $\operatorname{Re}(\lambda) \geq 0$ and $N-m$ eigenvalues $\lambda$ with $\operatorname{Re}(\lambda) \leq 0$.

Proof. The matrix $\mathbf{L}$ has all its off-diagonal elements -1 or 0 depending on whether there is a connection between the nodes associated with the associated column/row. The diagonal elements are the number of neighbors of the corresponding agent, and hence it is equal to the negative sum of the rest of the row elements [10]. From Geršgorin's theorem [11] it follows that the eigenvalues lie in the union of $N$ discs. The $i$ th disc has center $\beta_{i} L_{i i}$ and radius $\left|\beta_{i} L_{i i}\right|$. Following the logic of the proof for the Geršgorin theorem in [11], it is then possible to conclude that $m$ eigenvalues lie in the union of the discs defined by the positive elements of $\boldsymbol{\beta}$, and $N-m$ eigenvalues lie in the union of the discs defined by the negative elements of $\boldsymbol{\beta}$. To show this, write $\mathbf{B L}$ as the sum of a matrix $\mathbf{A}_{\mathrm{d}}$, containing only the diagonal elements of $\mathbf{B L}$, and a matrix $\mathbf{A}_{\text {od }}$, containing only the offdiagonal elements of BL. Then, for arbitrarily small positive values of $\epsilon$, the eigenvalues of $\mathbf{A}_{\mathrm{d}}+(1-\epsilon) \mathbf{A}_{\text {od }}$ are located in two disjoint regions, since the center of the discs is still $\beta_{i} L_{i i}$ but the radius is $(1-\epsilon)\left|\beta_{i} L_{i i}\right|$. Since the eigenvalues of a matrix are continuous functions of its entries, the eigenvalues located in the negative half-plane for $\epsilon>0$ can only go to zero as $\epsilon$ goes to zero, and they do not enter the positive plane. Likewise, the eigenvalues located in the positive halfplane do not enter into the negative half-plane as $\epsilon$ goes to zero.

\section{B. Circulant Graphs and Equal Weights}

In general, there is no known closed-form expression for the eigenvalues and eigenvectors of BL. For special classes of graphs and configurations of weights, however, this is possible.

Next, we present the analysis where all agents have the same weight, i.e., $\mathbf{B}=\alpha \mathbf{I}$, and the communication graph $\mathcal{G}$ is such that $\mathbf{L}$ is a circulant matrix. In this case, one can explicitly compute the eigenvectors and eigenvalues of the matrix BL. Recall that each row of a circulant matrix is equal 
to the row above it, with its elements shifted one position to the right such that the last element of the previous row becomes the first element of the current row. We call a graph with a circulant Laplacian $\mathbf{L}$ a circulant graph. A ring graph, as analyzed in [9], is an example of a circulant graph.

Lemma 3. Let $\mathbf{B}=\alpha \mathbf{I}$ and let $\mathcal{M} \subseteq\left\{1, \ldots,\left\lfloor\frac{N-1}{2}\right\rfloor\right\}$. Assume that there exists an edge between every two nodes $i$ and $j$ where $i \in \mathcal{V}$ and $j=((i+m-1)$ $\bmod N)+1$ with $m \in \mathcal{M}$. Then the eigenvectors of BL are $\mathbf{v}_{i}=\left[\omega_{i}^{0} \omega_{i}^{1} \ldots \omega_{i}^{N-1}\right]^{\mathrm{T}}$ with $\omega_{i}=\exp \left(\frac{2 \pi j i}{N}\right)$, and the corresponding eigenvalues are $\lambda_{i}=\alpha(2|\mathcal{M}|-$ $\left.2 \sum_{m \in \mathcal{M}} \cos \left(\frac{2 \pi}{N} i m\right)\right)$ for $i \in \mathcal{V}$.

Proof. Every agent is connected to $2|\mathcal{M}|$ other agents. Therefore the diagonal elements of $\mathbf{L}$ are equal to $2|\mathcal{M}|$. The off-diagonal elements of $\mathbf{L}$ in the $i$ th row and the $j$ th column are -1 if $j=((i+m-1) \bmod N)+1$ for $m \in \mathcal{M}$ and zero otherwise. Hence, $\mathbf{L}$ is a circulant matrix. The eigenvectors of a circulant matrix have the form $\mathbf{v}_{i}=\left[\omega_{i}^{0} \omega_{i}^{1} \ldots \omega_{i}^{N-1}\right]^{\mathrm{T}}$ with $\omega_{i}=\exp \left(\frac{2 \pi j i}{N}\right)$ for $i=1 \ldots N$ (see for instance [12]). It follows that these are also the eigenvectors of the matrix $\alpha \mathbf{L}$. From the first row of $\mathbf{L v}_{i}=\lambda_{i, \mathbf{L}} \mathbf{v}_{i}$, recalling the structure of $\mathbf{L}$ as described above, we get the expression $\lambda_{i, \mathbf{L}} \omega_{i}^{0}=$ $2|\mathcal{M}| \omega_{i}^{0}-\sum_{m \in \mathcal{M}}\left(\omega_{i}^{m}+\omega_{i}^{N-m}\right)$ which yields $\lambda_{i, \mathbf{L}}=$ $2|\mathcal{M}|-\sum_{m \in \mathcal{M}}\left(\exp \left(\frac{2 \pi j}{N} i m\right)+\exp \left(\frac{2 \pi j}{N} i(N-m)\right)\right)=$ $2|\mathcal{M}|-\sum_{m \in \mathcal{M}}^{m \in \mathcal{M}}\left(\exp \left(\frac{2 \pi \jmath}{N} i m\right)+\exp \left(\frac{2 \pi \jmath}{N} i(-m)\right)\right)=2|\mathcal{M}|-$ $2 \sum \cos \left(\frac{2 \pi}{N} i m\right)$. The eigenvalues of $\alpha \mathbf{L}$ are hence given by $\lambda_{i}=\alpha \lambda_{i, \mathbf{L}}$.

Lemma 3 gives the eigenvectors of $\mathbf{B L}$ as complex vectors. If we want to make the connection to the solutions of (3) using Theorem 1, we prefer a basis of real eigenvectors as the solutions $\mathbf{x}(t)$ are real. Therefore, we establish the following two lemmas, which together show how a complete basis of real eigenvectors can be constructed from the set of complex eigenvectors obtained by Lemma 3.

Lemma 4. Let BL be as in Lemma 3. Then, BL has one eigenvector $\mathbf{1} \in \mathbb{R}^{N}$. If $N$ is even, then $\mathrm{BL}$ has an eigenvector with its $k$ th element equal to $(-1)^{k-1}(k=$ $1, \ldots, N)$. The remaining eigenvectors of $\mathrm{BL}$ have linearly independent real and imaginary non-zero parts.

Proof. According to Lemma 3, the $k$ th element of $\mathbf{v}_{i}$ is $\omega_{i}^{k-1}=\exp \left(\frac{2 \pi j}{N} i(k-1)\right)$ for $k=1, \ldots, N$. This is a complex number of magnitude 1 and argument $(2 \pi \jmath / N) i(k-1)$. The real (resp. imaginary) parts of all elements of $\mathbf{v}$ are zero only if the argument is $n \pi$ (resp. $n \pi+\frac{\pi}{2}$ ) where $n \in \mathbb{Z}$. There are only two cases when this occurs. The first case is when $i=N$, which gives the eigenvector 1 . The other case is when $i=N / 2$, which exists only if $N$ is even. Then the $k$ th element of the eigenvector is given by $\exp \left(\frac{2 \pi \jmath}{N} \frac{N}{2}(k-1)\right)=\exp (\pi \jmath(k-1))=(-1)^{k-1}$. The real and imaginary parts of $\mathbf{v}$ are linearly independent since the first element of $\mathbf{v}$ is always 1 , which means that the first element of $\operatorname{Re}(\mathbf{v})$ is 1 and the first element of $\operatorname{Im}(\mathbf{v})$ is 0 .

There can only be $N$ linearly independent eigenvectors of BL. The next theorem gives a subset of eigenvectors, which (when the real and imaginary parts are considered separately) constitute a complete basis of linearly independent eigenvectors.

Lemma 5. The real and imaginary parts of the eigenvectors of BL for $i=\left\lceil\frac{N+1}{2}\right\rceil, \ldots, N-1$ span the same space as the real and imaginary parts of the eigenvectors of $\mathrm{BL}$ for $i=1, \ldots,\left\lfloor\frac{N-1}{2}\right\rfloor$.

Proof. Let $v_{i, m}=\omega_{i}^{m-1}$ be the $m$ th element of $\mathbf{v}_{i}$. Then for $\ell \in\left\{1, \ldots,\left\lfloor\frac{N-1}{2}\right\rfloor\right\}$ it follows that $\operatorname{Re}\left(v_{N-\ell, m}\right)=\operatorname{Re}\left(\omega_{N-\ell}^{m-1}\right)=\cos \left(\frac{2 \pi}{N}(m-1)(N-\ell)\right)=$ $\cos \left(\frac{2 \pi}{N}(m-1) \ell\right)=\operatorname{Re}\left(v_{\ell, m}\right)$. Similarly for the imaginary parts we have that $\operatorname{Im}\left(v_{N-\ell, m}\right)=\operatorname{Im}\left(\omega_{N-\ell}^{m-1}\right)=$ $\sin \left(\frac{2 \pi}{N}(m-1)(N-\ell)\right)=-\sin \left(\frac{2 \pi}{N}(m-1) \ell\right)=$ $-\operatorname{Im}\left(v_{\ell, m}\right)$.

Therefore, a complete base of eigenvectors for BL can be constructed from the real eigenvector(s) $\mathbf{v}_{N}, \mathbf{v}_{\frac{N}{2}}$ if $N$ is even, and from the real and imaginary parts of $\mathbf{v}_{i}$ for $i=1, \ldots,\left\lfloor\frac{N-1}{2}\right\rfloor$. Since $\mathbf{B L}$ is a real matrix, the imaginary and real parts of an eigenvector correspond to the same eigenvalue. We summarize the implications of Lemmas 3, 4 , and 5 in the following theorem:

Theorem 4. Let $\mathrm{BL}$ be as in Lemma 3. Then a basis of real eigenvectors of $\mathrm{BL}$ can be constructed by:

(a) One eigenvector $\mathbf{1} \in \mathbb{R}^{N}$ with eigenvalue 0 ,

(b) The eigenvectors $\left\{\operatorname{Re}\left(\mathbf{v}_{i}\right), \operatorname{Im}\left(\mathbf{v}_{i}\right) \mid i \in\left\{1, \ldots,\left\lfloor\frac{N-1}{2}\right\}\right\rfloor\right\}$ with $\mathbf{v}_{i}=\left[\omega_{i}^{0} \omega_{i}^{1} \ldots \omega_{i}^{N-1}\right]^{\mathrm{T}}$ and $\omega_{i}=\exp \left(\frac{2 \pi j i}{N}\right)$ with eigenvalue $\alpha\left(2|\mathcal{M}|-2 \sum_{m \in \mathcal{M}} \cos \left(\frac{2 \pi}{N} i m\right)\right)$,

(c) If $N$ is even, one eigenvector with its kth element equal to $(-1)^{k-1}(k=1, \ldots, N)$ and eigenvalue $\alpha(2|\mathcal{M}|-$ $\left.2 \sum_{m \in \mathcal{M}} \cos (\pi m)\right)$.

Together with Theorem 1, Theorem 4 provides a complete analytical solution of (3), which gives insight into the sustained oscillations exhibited by (3) if $\boldsymbol{\Gamma}=\mathbf{0}$. Similar results can be derived if all diagonal elements of $\mathbf{B}$ are such that all $\beta_{i}$ with even index $i$ are equal and all $\beta_{i}$ with odd index $i$ are also equal. The details are omitted owing to space constraints.

\section{DOUble InTEgRATOR DYNAMiCS}

In this section, we consider double integrator dynamics instead of single integrator dynamics for the individual agents. We introduce relative damping by the control law. The state of each agent $i$ is composed of the two-dimensional position vector $\mathbf{x}_{x, i} \in \mathbb{R}^{2}$ and the corresponding velocity vector $\dot{\mathbf{x}}_{x, i}=\mathbf{x}_{v, i} \in \mathbb{R}^{2}$. The control input for each agent is the acceleration, that is, $\dot{\mathbf{x}}_{v, i}=\mathbf{u}_{i}$. Furthermore, by defining the relative position measurements as $\mathbf{z}=\left(D^{\mathrm{T}} \otimes \mathbf{I}_{2}\right) \mathbf{x}_{x}$ 
we have $\dot{\mathbf{z}}=\left(D^{\mathrm{T}} \otimes \mathbf{I}_{2}\right) \mathbf{x}_{v}$, where, as before, we have defined the stack vectors $\mathbf{x}_{x}=\left[\begin{array}{lll}\mathbf{x}_{x, 1}^{\mathrm{T}} & \ldots & \mathbf{x}_{x, N}^{\mathrm{T}}\end{array}\right]^{\mathrm{T}} \in \mathbb{R}^{2 N}$, $\mathbf{x}_{v}=\left[\begin{array}{lll}\mathbf{x}_{v, 1}^{\mathrm{T}} \ldots & \mathbf{x}_{v, N}^{\mathrm{T}}\end{array}\right]^{\mathrm{T}} \in \mathbb{R}^{2 N}, \mathbf{u}=\left[\begin{array}{lll}\mathbf{u}_{1}^{\mathrm{T}} & \ldots & \mathbf{u}_{N}^{\mathrm{T}}\end{array}\right]^{\mathrm{T}} \in \mathbb{R}^{2 N}$ and $\mathbf{x}=\left[\mathbf{x}_{x}^{\mathrm{T}} \mathbf{x}_{v}^{\mathrm{T}}\right]^{\mathrm{T}} \in \mathbb{R}^{4 N}$.

The control input is given by a feedback law similar to (2), namely, $\mathbf{u}=\left(-\left(\mathbf{\Gamma} \mathbf{D} \otimes \mathbf{I}_{2}\right)+(\mathbf{B D} \otimes \mathbf{S})\right)(\mathbf{z}+\alpha \dot{\mathbf{z}})$, where $\boldsymbol{\Gamma}, \mathbf{B} \in \mathbb{R}^{N \times N}$ are diagonal matrices and $\alpha \in \mathbb{R}$ is the damping coefficient. With this feedback law, we get the overall dynamics

$$
\dot{\mathbf{x}}=\left[\begin{array}{cc}
\mathbf{0} & \mathbf{I} \\
\boldsymbol{\Theta} & \alpha \boldsymbol{\Theta}
\end{array}\right] \mathbf{x}=\boldsymbol{\Theta}_{\mathrm{d}} \mathbf{x} .
$$

where $\boldsymbol{\Theta}=-\left(\boldsymbol{\Gamma} \mathbf{L} \otimes \mathbf{I}_{2}\right)+(\mathbf{B L} \otimes \mathbf{S})$.

We can express the eigenvalues and eigenvectors of $\boldsymbol{\Theta}_{\mathrm{d}}$ in (5) in terms of those of $\Theta$. In the previous sections, we analyzed the eigenvalues and eigenvectors of matrices of this type. Hence, we can use the following theorem in order to apply the results from single integrator dynamics to double integrator dynamics.

Lemma 6. Let $\Theta_{\mathrm{d}}$ be as in (5) with $\alpha \geq 0$, and let $\tilde{\mathbf{v}}_{x}$ be an eigenvector of $\Theta$ with corresponding eigenvalue $\lambda$. Then $\tilde{\mathbf{v}}=\left[\tilde{\mathbf{v}}_{x}^{\mathrm{T}} \tilde{\lambda} \tilde{\mathbf{v}}_{x}^{\mathrm{T}}\right]^{\mathrm{T}}$ is an eigenvector of $\boldsymbol{\Theta}_{\mathrm{d}}$ with corresponding eigenvalue

$$
\tilde{\lambda}=\frac{\alpha \lambda \pm \sqrt{\alpha^{2} \lambda^{2}+4 \lambda}}{2} .
$$

Proof. Given the system dynamics in (5) the proof is similar to the proof of Theorem 6 in [13], and is therefore omitted.

Since $\lambda$ is complex valued, the connection between $\lambda$ and $\tilde{\lambda}$ is not trivial. As shown in [13], equation (6) also holds for double integrator consensus dynamics under a directed communication topology. From [13] we get the following sufficient condition for $\alpha$ to guarantee that $\tilde{\lambda}$ is in the lefthalf of the complex plane if $\lambda$ has negative real part.

Lemma 7. Let $\tilde{\lambda}$ as in (6) where $\alpha \geq 0$ and $\operatorname{Re}(\lambda)<0$. If $\alpha>\sqrt{-2 / \operatorname{Re}(\lambda)}>0$ then $\operatorname{Re}(\tilde{\lambda})<0$.

The proof of Lemma 7 is a slight modification of the proof of Theorem 6 in [13], and hence is omitted.

Assuming that $\boldsymbol{\Gamma}>0$, it follows from [7] that the eigenvalues of $\Theta$ have negative real part, and hence Theorem 6 and Lemma 7 imply that we can find $\alpha>0$, sufficiently large, such that all eigenvalues of the matrix $\Theta_{d}$ also have negative real part, except for two double eigenvalues in zero, which correspond to a uniform motion of the whole formation. In particular, Lemma 7 gives a sufficient condition on $\alpha$ for the system (5) to converge. This observation leads us to the main result of the this section.

\section{Theorem 5. The dynamic system defined in (5) asymptotically reaches consensus, i.e., $\lim _{t \rightarrow \infty}\left(\left\|\left[\mathbf{x}_{x, i}^{\mathrm{T}} \mathbf{x}_{v, i}^{\mathrm{T}}\right]^{\mathrm{T}}-\left[\mathbf{x}_{x, j}^{\mathrm{T}} \mathbf{x}_{v, j}^{\mathrm{T}}\right]^{\mathrm{T}}\right\|\right)=0$ for all $i, j \in \mathcal{V}$ if $\Gamma>0, \alpha>\sqrt{-2 / \operatorname{Re}(\lambda)}$ for all eigenvalues $\lambda$ of $\Theta$ with $\lambda \neq 0$, and $\Theta$ has two eigenvalues in zero.}

Proof. If $\lambda \neq 0$, we know from [7] that all non-zero eigenvalues of $\Theta$ have negative real part. From Lemma 6

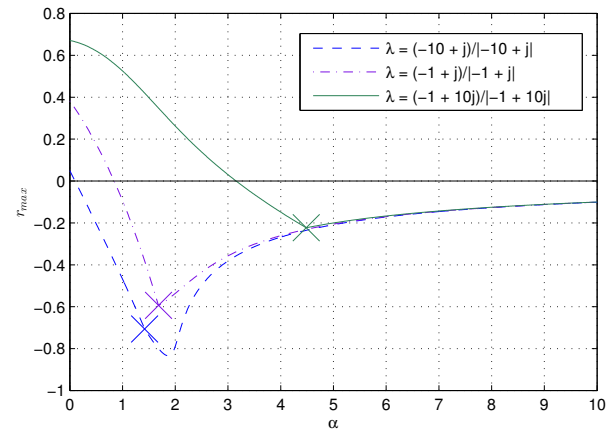

Fig. 1. Maximum real part of $\tilde{\lambda}=\frac{1}{2}\left(\alpha \lambda \pm \sqrt{\alpha^{2} \lambda^{2}+4 \lambda}\right)$ for three different $\lambda$ over different values of $\alpha$. A cross marks the point where $\alpha$ is according to Lemma 7 for that particular eigenvalue.

and 7, we can conclude that the condition $\alpha>\sqrt{-2 / \operatorname{Re}(\lambda)}$ ensures that all $\Theta_{\mathrm{d}}$ have negative real part. This means that the corresponding modes will converge to zero as time goes to infinity. Furthermore, we know from [7] that the null-space of $\boldsymbol{\Theta}$ is spanned by $\mathbf{v}_{c, 1}=\mathbf{1} \otimes[1,0]^{\mathrm{T}}$ and $\mathbf{v}_{c, 2}=\mathbf{1} \otimes[0,1]^{\mathrm{T}}$ (because (3) reaches consensus). These two vectors are mapped according to Lemma 6 into eigenvectors $\left[\mathbf{v}_{c, 1}^{\mathrm{T}} \mathbf{0}^{\mathrm{T}}\right]^{\mathrm{T}}$, $\left[\begin{array}{ll}\mathbf{v}_{c, 2}^{\mathrm{T}} & \mathbf{0}^{\mathrm{T}}\end{array}\right]^{\mathrm{T}}$ of $\boldsymbol{\Theta}_{\mathrm{d}}$ with double eigenvalue in zero, which correspond to modes $a_{1} t\left[\mathbf{v}_{c, 1}^{\mathrm{T}} \mathbf{0}^{\mathrm{T}}\right]^{\mathrm{T}}, a_{2} t\left[\mathbf{v}_{c, 1}^{\mathrm{T}} \mathbf{0}^{\mathrm{T}}\right]^{\mathrm{T}}$, where $t$ is time, and $a_{1}, a_{2}$ are coefficients depended on the initial conditions. Hence, $\lim _{t \rightarrow \infty}\left(\left|\left[\mathbf{x}_{x, i}^{\mathrm{T}} \mathbf{x}_{v, i}^{\mathrm{T}}\right]^{\mathrm{T}}-\left[\mathbf{x}_{x, j}^{\mathrm{T}} \mathbf{x}_{v, j}^{\mathrm{T}}\right]^{\mathrm{T}}\right|\right)=$ 0 for all $i, j \in \mathcal{V}$.

Given an eigenvalue $\lambda$ of $\boldsymbol{\Theta}$, we can numerically calculate the two values for $\tilde{\lambda}$ according to (6) for a range of values of $\alpha$. Figure 1 shows the real part of the two values of $\tilde{\lambda}$ with the larger real part, denoted by $r_{\max }$, resulting from three different choices of $\lambda$ and for different values of $\alpha$. The minimal value of $\alpha$ to ensure stability, according to Lemma 7, is marked with a cross. We see that $r_{\max }$ is positive for small $\alpha$, which means that the system diverges. Increasing $\alpha, r_{\max }$ drops and gets negative. It is easy to see from (6) that $r_{\max }$ has to be positive if the imaginary part of $\lambda$ is non-zero and $\alpha=0$. At some point $r_{\max }$ is smallest, which corresponds to fastest convergence. With higher values of $\alpha, r_{\max }$ asymptotically increases towards zero, which can be interpreted as the system response becoming slow due to damping. The magnitude of all three $\lambda \mathrm{s}$ in Figure 1 are normalized to one, so they only differ in terms of their phase angle. The smaller the phase angle, i.e., the larger the imaginary part, the larger $\alpha$ needs to be to ensure that $r_{\max }$ is negative. Also, the smaller the phase angle the larger the $r_{\max }$. For larger values of $\alpha$, there is not much of a difference. For smaller phase angles, the point with minimum $r_{\max }$ happens to coincide with the minimum $\alpha$ given by Lemma 7, whereas for values of $\lambda$ with the largest phase angle the minimum $r_{\max }$ is obtained for slightly larger $\alpha$.

\section{Simulations}

The first part of the numerical simulations illustrates Theorem 1 and Theorem 4. We let the communication graph 


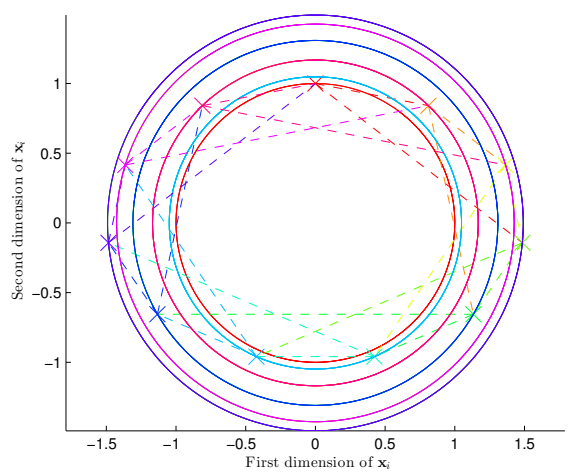

Fig. 2. Simulated trajectories of the agents in the plane with $\boldsymbol{\Gamma}=\mathbf{0}$ and $\mathbf{B}=\mathbf{I}$. The crosses represent the agents initial conditions. There are $N=11$ agents. The dashed lines indicate the edges in the communication graph. The communication graph $\mathcal{G}$ is as in Lemma 3 with $\mathcal{M}=\{1,3\}$.

$\mathcal{G}$ be as in Lemma 3 with $\mathcal{M}=\{1,3\}$. We use the weights $\boldsymbol{\Gamma}=\mathbf{0}$ (hence we expect periodic solutions) and $\mathbf{B}=\mathbf{I}$. We choose such initial conditions that only the four modes with smallest (in terms of magnitude) non-zero eigenvalue for BL are excited. According to Theorem 4, we have that $\lambda_{1}=4-2\left(\cos \left(\frac{2 \pi}{11} 1 \cdot 1\right)+\cos \left(\frac{2 \pi}{11} 1 \cdot 3\right)\right) \approx 2.60$ resulting in oscillations with period of length 2.41. The plane trajectories are shown in Figure 2. We can see that the trajectories indeed are circles. Furthermore, the period of the oscillations is indeed $T=2 \pi / 2.60 \approx 2.41$

In the second part of the numerical simulations, we present a simulation to illustrate the results of Section V. We choose a ring graph with $N=9$ agents having double integrator dynamics. The gain matrices are $\mathbf{B}=\boldsymbol{\Gamma}=\mathbf{I}$.

In the first simulation we choose the minimal sufficient $\alpha$ according to Lemma 7, which, in this case, happens to be the value that gives fastest convergence. In the top plot of Figure 3 we see that the agents converge to a consensus. For the second simulation, we set $\alpha$ to the value which is at the border of divergence. In the special case of a ring graph, two critical eigenvalues of the matrix $\mathrm{BL}$ are the same, and therefore two modes are associated to an eigenvalue with zero real part. The simulation results are shown in the lower plot of Figure 3. We see, that after some time sustained oscillations of the same frequency occur. These are caused by the two modes corresponding to the purely imaginary pair of eigenvalues.

\section{CONCLUSIONS AND Future WORK}

We have analyzed the distributed control strategy proposed in [7] with the focus on trajectories of sustained oscillations. We have shown the relationship of the system trajectories with the eigenvalues and eigenvectors of a scaled graph Laplacian. Furthermore, we have provided a number of results on such eigenvalues and eigenvectors for the general and for a selected, specific case. We have also extended the proposed control strategy to double-integrator dynamics. We have provided a sufficient condition for convergence to a
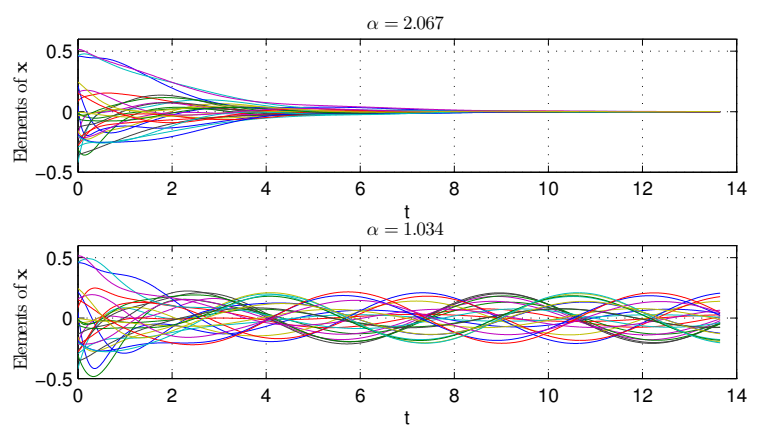

Fig. 3. Simulations of the extended consensus algorithm for double integrator dynamics. In the upper plot, the damping factor $\alpha$ was chosen such that convergence is the fastest. On the lower plot, it was set to the limit of convergence. The plots show the elements of $\mathbf{x}(t)$.

consensus point and analyzed the influence of the damping coefficient. Finally, we have illustrated the theoretical results via numerical simulations. The results of this paper can be used to design periodic trajectories for the agents having specific properties, that is, in contrast to the results of [7], it addresses the synthesis question. Future work will investigate the eigenvalues and eigenvectors of additional special configurations and different agent dynamics.

\section{REFERENCES}

[1] R. Olfati-Saber and R. Murray, "Consensus problems in networks of agents with switching topology and time-delays," IEEE Transactions on Automatic Control, vol. 49, no. 9, pp. 1520 - 1533, Sept. 2004

[2] J. Cortés, "Distributed algorithms for reaching consensus on general functions," Automatica, vol. 44, no. 3, pp. 726-737, Mar. 2008.

[3] Y. Q. Chen and Z. Wang, "Formation control: a review and a new consideration," Intelligent Robots and Systems, 2005. (IROS 2005), pp. 3181-3186, Aug. 2005.

[4] B. Anderson, B. Fidan, C. Yu, and D. Walle, "UAV formation control: Theory and application," in Recent Advances in Learning and Control, ser. Lecture Notes in Control and Information Sciences, V. Blondel, S. Boyd, and H. Kimura, Eds. Springer London, 2008, vol. 371, pp. 15-33.

[5] T.-H. Kim and T. Sugie, "Cooperative control for target-capturing task based on a cyclic pursuit strategy." Automatica, vol. 43, no. 8, pp. 1426-1431, 2007.

[6] R. Sepulchre, D. A. Paley, S. Member, and N. E. Leonard, "Stabilization of planar collective motion: all-to-all communication," IEEE Transactions on Automatic Control, vol. 52, pp. 811-824, 2007.

[7] P. Tsiotras and L. I. R. Castro, "A note on the consensus protocol with some applications to agent orbit pattern generation," in Distributed Autonomous Robotic Systems, ser. Springer Tracts in Advanced Robotics, A. Martinoli, F. Mondada, N. Correll, G. Mermoud, M. Egerstedt, M. A. Hsieh, L. E. Parker, and K. Støy, Eds. Springer Berlin Heidelberg, 2013, vol. 83, pp. 345-358.

[8] P. Tsiotras and L. I. R. Castro, Controls and Art, ser. Springer Lectures Notes on Computer Science. Springer, 2014, ch. The Artistic Geometry of Consensus Protocols, pp. 129-153.

[9] W. Ren, "Collective motion from consensus with Cartesian coordinate coupling," IEEE Transactions on Automatic Control, vol. 54, no. 6 , pp. 1330-1335, June 2009.

[10] C. Godsil and G. Royle, Algebraic Graph Theory. Springer, Apr. 2001.

[11] R. A. Horn and C. R. Johnson, Matrix Analysis. Cambridge University Press, 1990.

[12] P. J. Davis, Circulant Matrices, 2nd ed. New York, NY: AMS Chelsea Publishing., 1994.

[13] S. Roy, A. Saberi, and K. Herlugson, "Formation and alignment of distributed sensing agents with double-integrator dynamics and actuator saturation," Sensor Network Applications, 2004. 